\title{
Bicipital origin and the course of the plantaris muscle
}

\author{
Yijin Heo ${ }^{1}$, Hyemin Lee ${ }^{2}$, Seung-Jun Hwang ${ }^{1,2}$ \\ ${ }^{1}$ Department of Anatomy, College of Medicine, University of Ulsan, Seoul, ${ }^{2}$ Clinical Anatomy Education Center, Asan Medical Center, Seoul, Korea
}

\begin{abstract}
The plantaris muscle (PM) has a small fusiform muscle belly and a long slender tendon sandwiched between the soleus (SM) and gastrocnemius muscle (GM). During routine dissection for research, an additional PM in the popliteal region of a 75-year-old Korean female was discovered. Two distinct PMs were present, the superior PM (sPM) and inferior PM (iPM). While the sPM originates from the lower lateral supracondylar ridge and the knee capsule, the iPM originates from the femoral condyle and sPM tendon splitting into two parts at the distal belly. The lateral side of the iPM tendon travels between GM and SM and ends at the calcaneal tendon. sPM and the medial side of the iPM tendon run along with the sPM tendon and inserts at the fascia at the inner surface of proximal $1 / 3$ of the medial head of GM. This case report introduces a new variation of the PM that should be taken into consideration.
\end{abstract}

Key words: Plantaris muscle, Plantaris tendon, Muscle belly, Gastrocnemius muscle, Anatomic variation

Received April 28, 2021; Accepted May 7, 2021

\section{Introduction}

The plantaris muscle (PM) is about 7 to $13 \mathrm{~cm}$ long and has a short spindle-shaped muscle belly originating from the lateral supracondylar ridge of the femur above the lateral head of gastrocnemius muscle (GM) and the knee capsule [14]. It also has a long slender tendon typically traveling inferomedially and ends at the calcaneal tuberosity along with the calcaneal tendon [5]. The PM is sandwiched between the GM and soleus muscle (SM) and innervated by the tibial nerve acting as a weak flexor of the knee and ankle [6]. The PM is absent in $7 \%$ to $20 \%$ of the general population, and many studies have reported it to be highly variable in its course and both the origin and insertion [1, 2, 6-10]. Although it has been considered a vestigial muscle, recent studies now

\section{Corresponding author:}

Seung-Jun Hwang (iD)

Department of Anatomy, College of Medicine, University of Ulsan, Seoul 05505, Korea

E-mail: sjhwang@amc.seoul.kr consider PM as a proprioceptive organ due to its high density of muscle spindles [7]. This case report introduces a unique and rare variation of the PM having two distinct bellies and tendons.

\section{Case Report}

During routine dissection of the popliteal area for research, a highly rare and unique variation having an additional PM was identified in the right-side leg of a 75-year-old Korean female formalin-fixed cadaver. The dissection was done carefully by a skilled anatomist and technician following the detailed and specified protocol by Olewnik et al. [3]. There were no signs of previous surgery or injury in the popliteal region, and there was no homologic variation on the opposite side.

When the PM was exposed, we found two distinct PMs with their own muscle bellies and tendons. The superior PM (sPM) originates from the lower lateral supracondylar ridge and the knee capsule. The inferior PM (iPM) originated from the knee joint capsule and the lateral head of GM, indirectly attached to the lateral femoral condyle and sPM tendon. It 

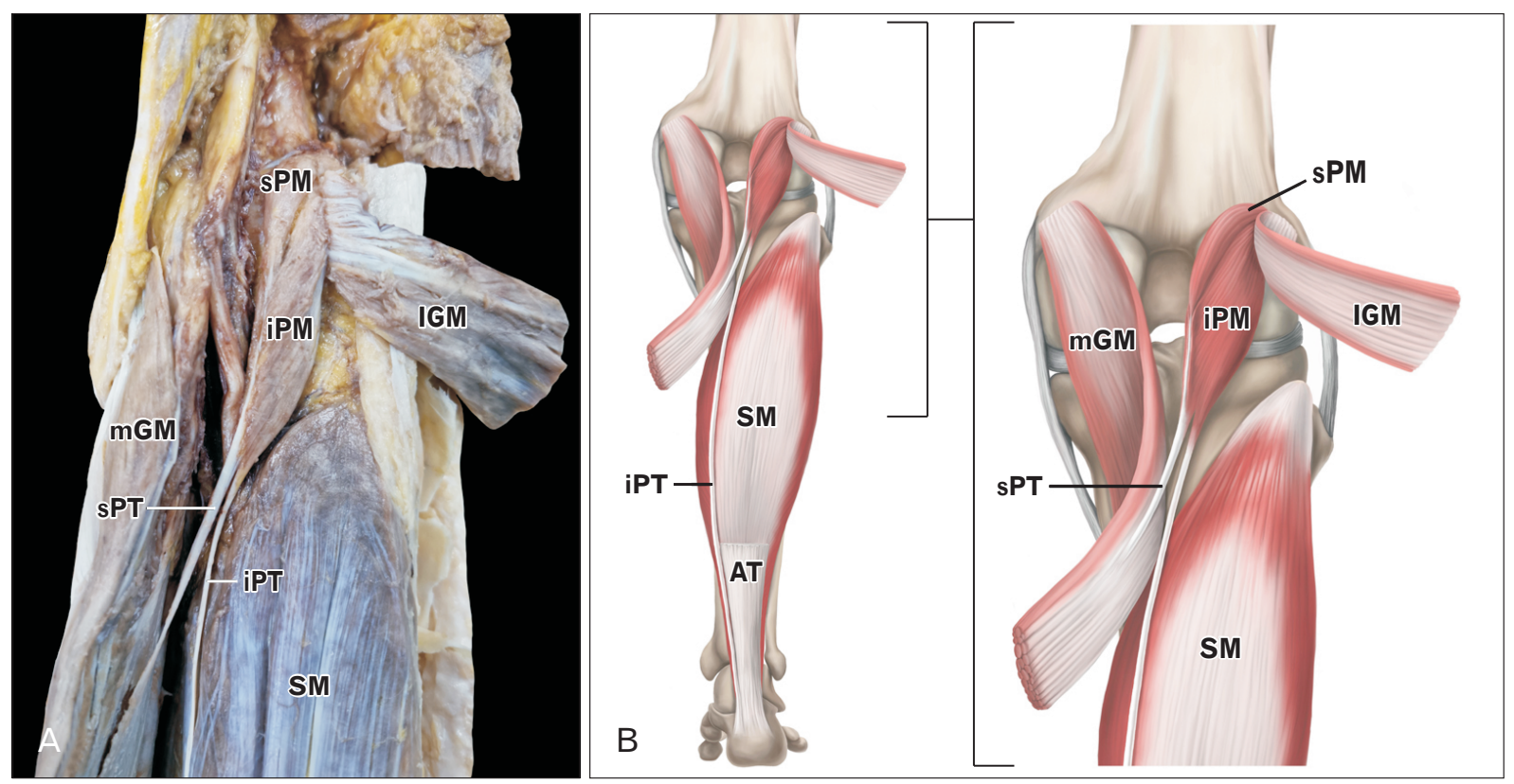

Fig. 1. Posterior view of the lower right leg. (A) The course of additional plantaris muscle/tendon unit. (B) Schematic drawing of plantaris muscle/tendon unit. AT, Achilles tendon (cut); iPM, inferior plantaris muscle; iPT, inferior plantaris tendon; lGM, lateral head of gastrocnemius muscle (cut); mGM, medial head of gastrocnemius muscle (cut); SM, soleus muscle (cut); sPM, superior plantaris muscle; sPT, superior plantaris tendon.

Table 1. Measurements of sPM and iPM

\begin{tabular}{ccccc}
\hline Type of PM & $\begin{array}{c}\text { Belly length } \\
(\mathrm{cm})\end{array}$ & $\begin{array}{c}\text { Belly width } \\
(\mathrm{cm})\end{array}$ & $\begin{array}{c}\text { MTJ width } \\
(\mathrm{cm})\end{array}$ & $\begin{array}{c}\text { Tendon length } \\
(\mathrm{cm})\end{array}$ \\
\hline sPM & 7.27 & 0.82 & 0.32 & 5.28 \\
iPM & 6.41 & 1.33 & 0.17 & 30.85 \\
\hline
\end{tabular}

iPM, inferior plantaris muscle; MTJ, myotendinous junction; sPM, superior plantaris muscle.

splits into two parts, the medial and lateral side at the distal belly. The lateral side of the iPM tendon travels inferomedially between GM and SM and is eventually inserted at the calcaneal tuberosity along with the calcaneal tendon without spreading out in a fan shape. The medial side of iPM merges with the sPM tendon, and its tendon travels along the iPM tendon until proximal $1 / 3$ of the medial head of GM (mGM). Then, the sPM tendon is inserted into the fascia at the inner surface of mGM (Fig. 1). The measurements of sPM and iPM are presented in Table 1.

\section{Discussion}

The PM has a small spindle-shaped muscle belly and a long slender tendon $[8,9]$. It typically originates from the lateral supracondylar ridge of the femur above the lateral head of GM and inserts at calcaneal tuberosity $[1,2,4,5]$. Its tendon travels inferomedially between GM and SM, which comprises the triceps surae muscle group working as a flexor of the knee and ankle along with PM [6]. It has been considered a vestigial muscle. However, recent studies consider PM a proprioceptive organ due to its high density of muscle spindles [7].

The PM is a biarticular muscle which makes it subject to rupture [2]. Most of the tears occur at the level of the myotendinous junction (MTJ) and muscle belly [11]. Injuries can occur solely or simultaneously with SM and GM, which is referred to as "Tennis leg." It is a common sports-associated injury, usually injured when the knee is excessively extended and the ankle is forced dorsiflexion [2]. Also, recent reports emphasize PM's potential intervention in the mid-portion tendinopathy of the Achilles tendon [3]. Moreover, the morphological features of the plantaris tendon make it a suitable source for tendon graft in surgical practice $[2,12]$.

The PM is absent in $7 \%-20 \%$ of the general population, and many studies have reported it to be highly variable in both the origin and insertion or even its course $[1,7]$. Some of them even reported it with two or more muscle bellies or tendons [11, 13]. Herzog [14] determined the prevalence of a new accessory PM. Out of 1,000 patients, the accessory PM was detected in 63 patients (6.3\%). 
Park et al. [13] reported an interesting variation of PM comparable to our case recently. They found PM having the same insertion site as SPM in our case on an 89-year-old Korean male leg. The difference was that PM was present as a single muscle, not double.

Rana et al. [15] reported double PM, similar to our case. They found two muscle bellies and tendons, the inner and outer belly. The tendon of the inner belly had a typical course while the tendon of the outer belly fused with the lateral side of the common tendon of GM and SM little below the middle of the leg, the tendons belonging to each belly. However, the variation they discovered bellies having their own tendons was quite different from our case.

While many studies have reported different kinds of unique variations of PM having accessory bellies or insertions as mentioned above, to the author's knowledge, none of them has ever reported an identical variation introduced in this case before (searched on PubMed, Embase, Cochrane Library, CINAHL, google scholar). Also, the width of the sPM MTJ and tendon, the additional PM were wider than the iPM, which distinguishes our case from others. This case report introduces a new type of PM that deserves consideration. When dealing with patients with posterior knee pain or harvesting tendon donors, understanding the normal anatomy and diverse variations of PM may be useful.

\section{ORCID}

Yijin Heo: https://orcid.org/0000-0003-1207-5678

Hyemin Lee: https://orcid.org/0000-0003-2130-3353

Seung-Jun Hwang:

https://orcid.org/0000-0002-4345-9773

\section{Author Contributions}

Conceptualization: YH, HL, SJH. Data acquisition: YH, HL. Data analysis or interpretation: YH. Drafting of the manuscript: YH. Critical revision of the manuscript: SJH. Approval of the final version of the manuscript: all authors.

\section{Conflicts of Interest}

No potential conflict of interest relevant to this article was reported.

\section{Acknowledgements}

The authors wish to express their gratitude to Yeonju Kim for illustration and Taejin Jung for data collection.

\section{References}

1. Olewnik Ł, Zielinska N, Karauda P, Tubbs RS, Polguj M. A three-headed plantaris muscle: evidence that the plantaris is not a vestigial muscle? Surg Radiol Anat 2020;42:1189-93.

2. Harwin JR, Richardson ML. "Tennis leg”: gastrocnemius injury is a far more common cause than plantaris rupture. Radiol Case Rep 2016;12:120-3.

3. Olewnik Ł, Wysiadecki G, Podgórski M, Polguj M, Topol M. The plantaris muscle tendon and its relationship with the Achilles tendinopathy. Biomed Res Int 2018;2018:9623579.

4. Helms CA, Fritz RC, Garvin GJ. Plantaris muscle injury: evaluation with MR imaging. Radiology 1995;195:201-3.

5. Olewnik Ł, Wysiadecki G, Polguj M, Topol M. Anatomic study suggests that the morphology of the plantaris tendon may be related to Achilles tendonitis. Surg Radiol Anat 2017;39:69-75.

6. Hadimani GA, Bagoji I. Accessory belly of the plantaris muscle and its clinical relevance. South East Asia J Med Sci 2018;2:5-6.

7. Kurtys K, Gonera B, Olewnik Ł, Karauda P, Tubbs RS, Polguj $\mathrm{M}$. Is the plantaris muscle the most undefined human skeletal muscle? Anat Sci Int 2020 Nov 7 [Epub]. https://doi.org/10.1007/ s12565-020-00586-4.

8. Olewnik Ł, Podgórski M, Polguj M, Topol M. The plantaris muscle - rare relations to the neurovascular bundle in the popliteal fossa. Folia Morphol (Warsz) 2018;77:785-8.

9. Olewnik Ł, Wysiadecki G, Polguj M, Topol M. The report on the co-occurrence of two different rare anatomic variations of the plantaris muscle tendon on both sides of an individual. Folia Morphol (Warsz) 2017;76:331-3.

10. Aragao JA, Reis FP, Guerra DR, Cabral RH. The occurrence of the plantaris muscle and its muscle-tendon relationship in adult human cadavers. Int J Morphol 2010;28:255-8.

11. Olewnik Ł, Kurtys K, Gonera B, Podgórski M, Sibiński M, Polguj M. Proposal for a new classification of plantaris muscle origin and its potential effect on the knee joint. Ann Anat 2020;231:151506.

12. Rohilla S, Jain N, Yadav R. Plantaris rupture: why is it important? BMJ Case Rep 2013;2013:bcr2012007840.

13. Park KR, Cho J, Choi YJ, Kim D, Kwon HW, Lee M, Yoon KH, Park J. Complex variations of plantaris muscle origin, course and insertion: a cadaveric case report. Anat Biol Anthropol 2020;33:143-7.

14. Herzog RJ. Accessory plantaris muscle: anatomy and prevalence. HSS J 2011;7:52-6.

15. Rana KK, Das S, Verma R. Double plantaris muscle: a cadaveric study with clinical importance. Int J Morphol 2006;24:495-8. 